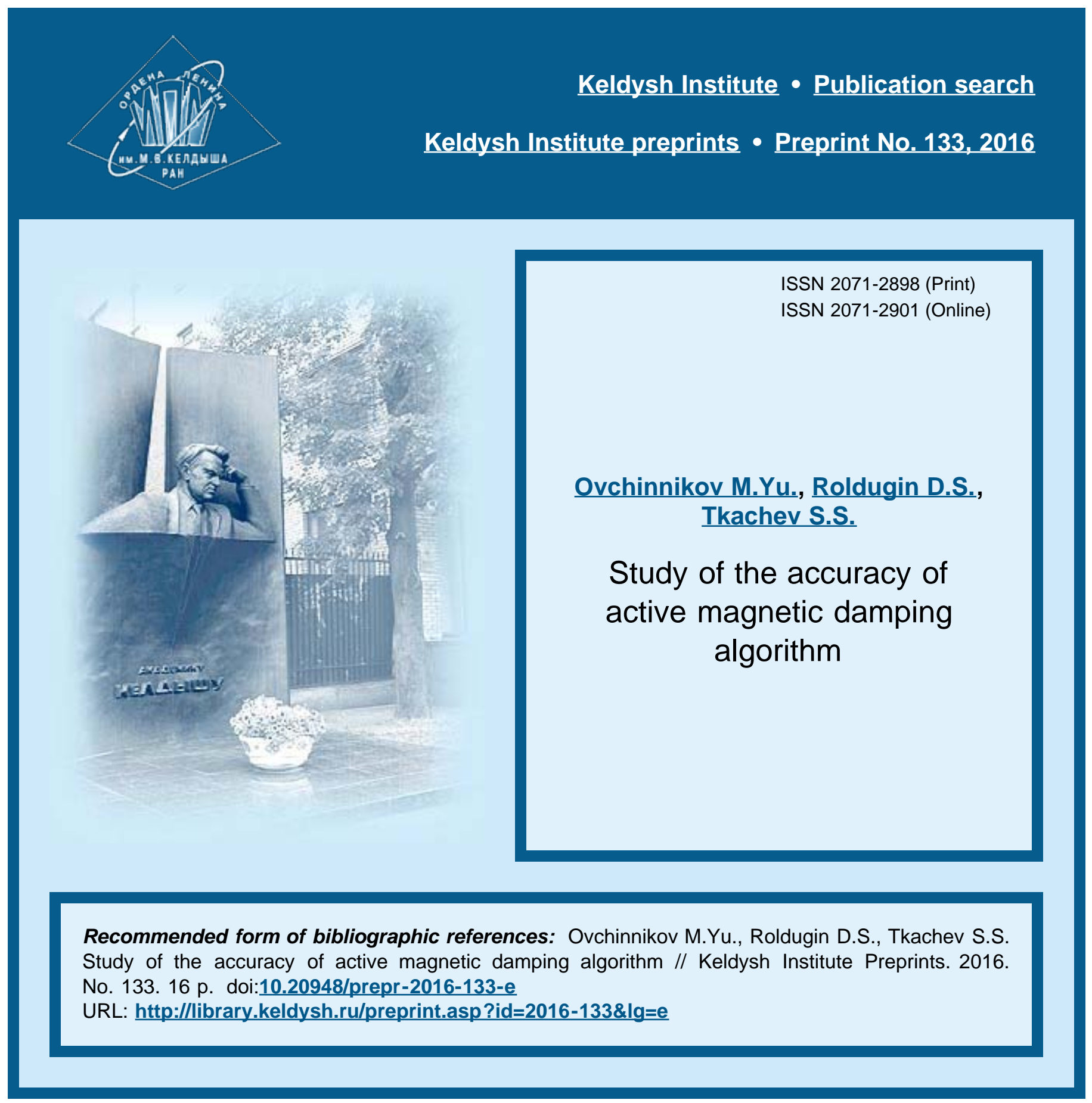




\author{
Ордена Ленина \\ ИНСТИТУТ ПРИКЛАДНОЙ МАТЕМАТИКИ \\ имени М.В. Келдыша \\ Российской академии нау
}

M.Yu. Ovchinnikov, D.S. Roldugin, S.S. Tkachev

\title{
Study of the accuracy of active magnetic damping algorithm
}




\section{М.Ю. Овчинников, Д.С. Ролдугин, С.С. Ткачев}

Исследование точности алгоритма активного магнитного демпфирования

Рассматривается спутник, оснащенный магнитной системой ориентации, реализующей алгоритм демпфирования угловой скорости «-Bdot». Исследуется поведение спутника в конце переходных процессов. Показано, что спутник медленно вращается вокруг оси максимального момента инерции, найдена величина скорости вращения. Исследуется точность ориентации спутника в дипольной модели геомагнитного поля. Проводится численное моделирование.

Ключевые слова: магнитная система ориентации, демпфирование, Bdot

\section{Mikhail Ovchinnikov, Dmitry Roldugin, Stepan Tkachev}

Study of the accuracy of active magnetic damping algorithm

Attitude motion of a satellite equipped with an active magnetic attitude control system is considered. Control system implements «-Bdot» damping algorithm. Satellite behavior is analyzed in a steady-state motion. Slow spinning around the principal axis of maximum inertia is proven, angular velocity is found. Attitude accuracy in dipole geomagnetic field model is studied. Numerical analysis is carried out.

Key words: magnetic attitude control system, damping, Bdot

The work was supported by RFBR grants № 15-31-20058 and 16-01-00634.

\section{Contents}

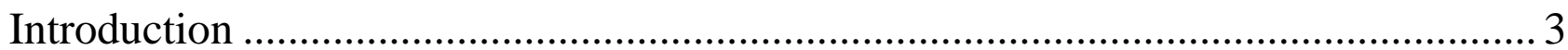

1. Equations of motion and geomagnetic field models ............................................. 3

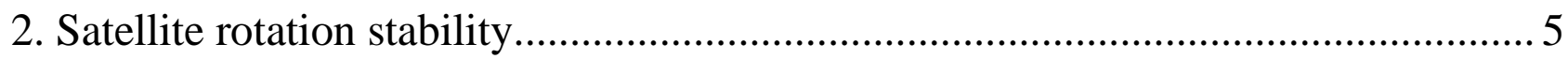

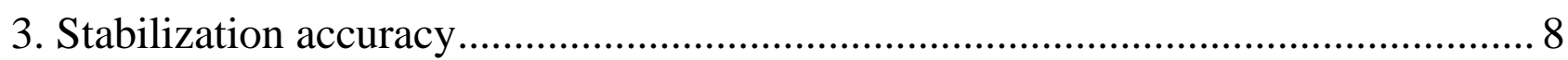

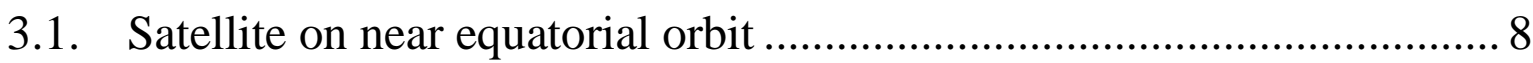

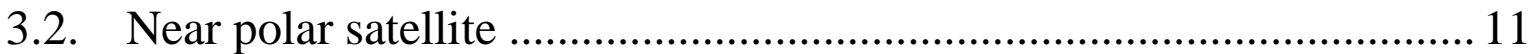

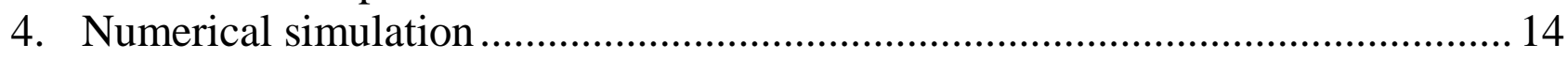

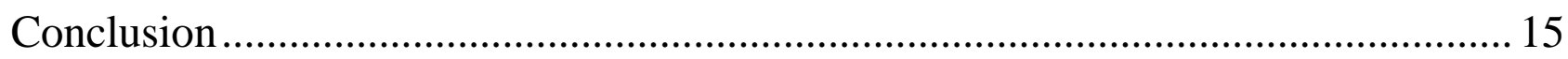

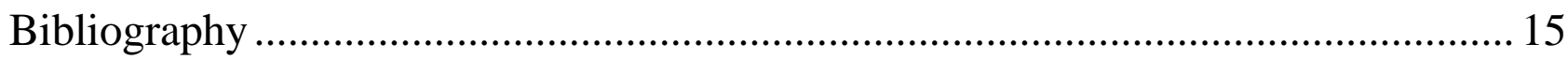

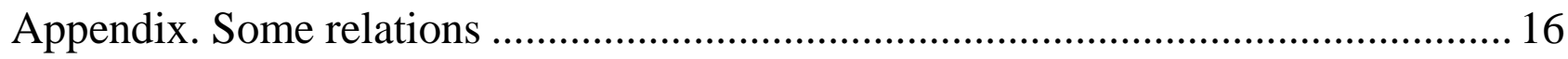




\section{Introduction}

Magnetic attitude control system is implemented on almost every modern small satellite. Even big and complex spacecraft utilize this system quite frequently. Magnetic control system has two main tasks: angular velocity damping and reaction wheel angular momentum unload. This work is devoted to the first problem. Attitude acquisition phase was considered by authors in [1,2] under fast rotating satellite assumption. In this paper satellite angular velocity is comparable with the orbital one. This attitude mode is relevant due to the most used magnetic damping algorithm «-Bdot» [3,4]. Its name implies that the control dipole moment is opposite to the geomagnetic induction vector change. Satellite acquires approximate rotation information with respect to geomagnetic field. Control system should spin satellite in the opposite direction effectively cancelling the rotation. However geomagnetic induction vector motion with respect to the satellite is due to its vector rotation in inertial space also. So the satellite should end up spinning with approximately double orbital angular velocity. Here this rotation is proven to be about the principal axis of the maximum moment of inertia. Spinning velocity value is refined, satellite attitude is assessed.

\section{Equations of motion and geomagnetic field models}

The satellite is considered to be a rigid body. Attitude is maintained using three orthogonal magnetorquers. Actual attitude is available without any error. Four reference frames are used:

Inertial reference frame $O_{a} Y_{1} Y_{2} Y_{3}, O_{a}$ is Earth's center, $O_{a} Y_{3}$ is directed along Earth's spin axis, $O_{a} Y_{l}$ lies in the equatorial plane and is directed to the ascending node, $O_{a} Y_{2}$ is directed so the system to be a right handed.

Inertial reference frame $O_{a} Z_{1} Z_{2} Z_{3}$ is got from frame $O_{a} Y_{1} Y_{2} Y_{3}$ turning by angle $\Theta$ about $O_{a} Y_{l}$. This angle is a function of orbit inclination. It is defined with averaged geomagnetic field model.

Inertial reference frame $O_{a} S_{1} S_{2} S_{3}$ is tied to satellite's orbit. $O_{a} S_{3}$ is normal to the orbital plane, $O_{a} S_{1}$ is directed to the ascending node, $O_{a} S_{2}$ is directed so the system to be a right handed. Frame $O_{a} S_{1} S_{2} S_{3}$ attitude with respect to $O_{a} Y_{1} Y_{2} Y_{3}$ is defined with rotation by angle $i$ (inclination) about $O_{a} Y_{1}$, rotation from $O_{a} S_{1} S_{2} S_{3}$ to $O_{a} Z_{1} Z_{2} Z_{3}$ is defined with angle $\Theta-i$ about $O_{a} S_{1}$. Inertial reference frames are depicted in fig. 1. 


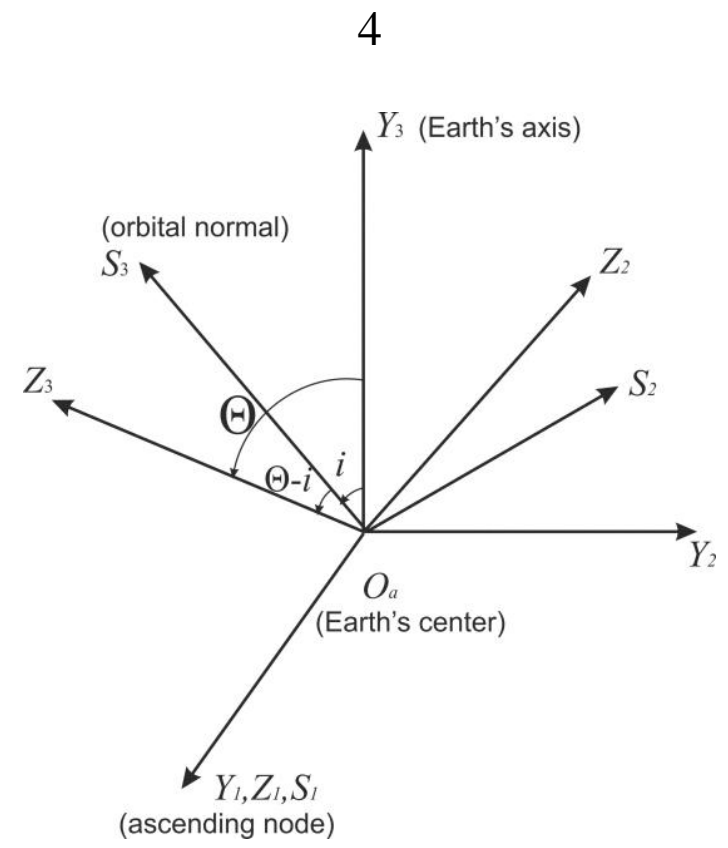

Fig. 1. Inertial reference frames

$O x_{1} x_{2} x_{3}$ is bound reference with axes directed along the principal axes of inertia.

Satellite attitude with respect to any inertial frame is defined using angles $\alpha, \beta, \gamma$ (rotation sequence 3-1-2) and absolute angular velocity components is bound reference frame $\left(\omega_{1}, \omega_{2}, \omega_{3}\right)$. Quaternions are used for numerical simulation. Direction cosines matrix is

$\mathbf{A}=\left(\begin{array}{ccc}\cos \alpha \cos \gamma-\sin \alpha \sin \beta \sin \gamma & \sin \alpha \cos \gamma+\cos \alpha \sin \beta \sin \gamma & -\cos \beta \sin \gamma \\ -\sin \alpha \cos \beta & \cos \alpha \cos \beta & \sin \beta \\ \cos \alpha \sin \gamma+\sin \alpha \sin \beta \cos \gamma & \sin \alpha \sin \gamma-\cos \alpha \sin \beta \cos \gamma & \cos \beta \cos \gamma\end{array}\right)$.

Dynamical equations of the satellite motion $\mathrm{e}$ with inertia tensor $\mathbf{J}=\operatorname{diag}(A, B, C)$ are

$\mathbf{J} \frac{d \boldsymbol{\omega}}{d t}+\boldsymbol{\omega} \times \mathbf{J} \boldsymbol{\omega}=\mathbf{M}$

where $\mathbf{M}$ is control magnetic torque.

These equations are complemented with kinematics

$\frac{d \alpha}{d t}=\frac{1}{\cos \beta}\left(-\omega_{1} \sin \gamma+\omega_{3} \cos \gamma\right)$,

$\frac{d \beta}{d t}=\omega_{1} \cos \gamma+\omega_{3} \sin \gamma$,

$\frac{d \gamma}{d t}=\omega_{2}+\operatorname{tg} \beta\left(\omega_{1} \sin \gamma-\omega_{3} \cos \gamma\right)$. 
Geomagnetic field is represented [5] with right dipole model and the averaged model (simplified dipole model). Right dipole implies Earth's field to be of the dipole $\mathbf{k}=(0,0,-1)$ in $O_{a} Y_{1} Y_{2} Y_{3}$ frame. Induction vector in frame $O_{a} S_{1} S_{2} S_{3}$ is

$\mathbf{B}_{S}=\frac{\mu_{e}}{r^{3}}\left(\begin{array}{c}-3 / 2 \sin 2 u \sin i \\ -3 \sin ^{2} u \sin i+\sin i \\ \cos i\end{array}\right)$

where $u$ is argument of latitude; $\mu_{e}=\mu_{0} \mu_{m} / 4 \pi, \mu_{m}$ is Earth's dipole strength (currently $\mu_{e}=7.7245 \cdot 10^{6} \mathrm{~T} \cdot \mathrm{km}^{3}$ ), $\mu_{0}=4 \pi \cdot 10^{-7} \mathrm{~kg} \cdot \mathrm{m} \cdot \mathrm{A}^{-2} \cdot \mathrm{s}^{-2}$ is magnetic constant.

Simplified dipole model provides the most compact field representation in inertial space. Induction vector has constant length and uniformly moves on the circular cone. Induction vector in frame $O_{a} Z_{1} Z_{2} Z_{3}$ is

$\mathbf{B}_{Z}=B_{0}\left(\begin{array}{c}-\sin \Theta \sin 2 u \\ \sin \Theta \cos 2 u \\ \cos \Theta\end{array}\right)$

where $B_{0}=\left(1+\sqrt{1+3 \sin ^{2} i}\right) \mu_{e} / 2 r^{3}$, cone half-opening angle is

$\operatorname{tg} \Theta=\frac{3 \sin 2 i}{2\left(1-3 \sin ^{2} i+\sqrt{1+3 \sin ^{2} i}\right)}$.

Satellite is subjected to «-Bdot» control. Dipole moment of magnetorquers is given by

$\mathbf{m}=-k \frac{d \mathbf{B}_{x}}{d t}$.

\section{Satellite rotation stability}

Here simplified dipole model is used. Satellite motion is considered in $O_{a} Z_{1} Z_{2} Z_{3}$ frame. Control dipole moment of magnetorquers is

$$
\mathbf{m}=\mathbf{m}_{1}+\mathbf{m}_{2}=k \boldsymbol{\omega} \times \mathbf{A B}_{Z}-k \mathbf{A} \frac{d \mathbf{B}_{Z}}{d t} .
$$

Satellite angular velocity is always considered fast when «-Bdot» algorithm is studied, $\boldsymbol{\omega} \gg \boldsymbol{\omega}_{0}$. In this case second term in (2.1) may be omitted. If only first part in (2.1) is implemented satellite angular velocity is asymptotically damped to zero. If second term is preserved angular velocity is damped approximately to double orbital velocity. In the end of damping process second term becomes of the same order as the first and cannot be omitted. 
Suppose the satellite is spun about its third axis and find conditions providing the existence and stability of the motion $\beta=\gamma=0, \omega_{1}=\omega_{2}=0$. Control dipole moment is this case is

$\mathbf{m}=k \sin \Theta\left(\begin{array}{c}-\omega_{3} \cos (\alpha-2 u)+2 \omega_{0} \cos (\alpha-2 u) \\ \omega_{3} \sin (\alpha-2 u)-2 \omega_{0} \sin (\alpha-2 u) \\ 0\end{array}\right)$.

This implies $\omega_{3}=2 \omega_{0}$ and $\alpha=2 u+\alpha_{0}$. The satellite and geomagnetic induction vector rotation rates are exactly the same. Suppose $\alpha_{0}=0$ and obtain linearized equations in the vicinity of motion $\alpha=2 u, \beta=\gamma=0, \omega_{1}=\omega_{2}=0, \omega_{3}=2 \omega_{0}$. Direction cosines matrix is

$\mathbf{A}=\left(\begin{array}{ccc}\cos 2 u-\alpha \sin 2 u & \sin 2 u+\alpha \cos 2 u & -\gamma \\ -\sin 2 u-\alpha \cos 2 u & \cos 2 u-\alpha \sin 2 u & \beta \\ \gamma \cos 2 u+\beta \sin 2 u & \gamma \sin 2 u-\beta \cos 2 u & 1\end{array}\right)$,

geomagnetic induction vector in bound frame

$\mathbf{B}_{x}=B_{0}\left(\begin{array}{c}\alpha \sin \Theta-\gamma \cos \Theta \\ \sin \Theta+\beta \cos \Theta \\ -\beta \sin \Theta+\cos \Theta\end{array}\right)$,

control dipole moments components are

$\mathbf{m}_{1}=k B_{0}\left(\begin{array}{c}\omega_{2} \cos \Theta-\omega_{3} \sin \Theta-2 \omega_{0} \sin \Theta-2 \omega_{0} \cos \Theta \beta \\ 2 \omega_{0} \sin \Theta \alpha-2 \omega_{0} \cos \Theta \gamma-\omega_{1} \cos \Theta \\ \omega_{1} \sin \Theta\end{array}\right), \mathbf{m}_{2}=2 \omega_{0} k B_{0} \sin \Theta\left(\begin{array}{c}1 \\ -\alpha \\ \gamma\end{array}\right)$.

Dimensionless linearized equations of motion are

$\dot{\omega}_{1}=2 \lambda_{A} \omega_{2}-\theta_{A} \varepsilon\left(\omega_{1}+2 \gamma\right)$,

$\dot{\omega}_{2}=2 \lambda_{B} \omega_{1}+\theta_{B} \varepsilon\left(-\omega_{2} \cos ^{2} \Theta+\omega_{3} \sin \Theta \cos \Theta+2 \cos ^{2} \Theta \beta\right)$,

$\dot{\omega}_{3}=\varepsilon\left(\omega_{2} \cos \Theta \sin \Theta-\omega_{3} \sin ^{2} \Theta-2 \sin \Theta \cos \Theta \beta\right)$,

$\dot{\alpha}=\omega_{3}, \quad \dot{\beta}=\omega_{1}+2 \gamma, \quad \dot{\gamma}=\omega_{2}-2 \beta$.

Here argument of latitude is used instead of time, angular velocity is referred to the orbital one, $\varepsilon=k B_{0}^{2} / C \omega_{0}, \quad \theta_{A}=C / A, \quad \theta_{B}=C / B, \quad \lambda_{A}=(B-C) / A$, $\lambda_{B}=(C-A) / B$. Equation for $\alpha$ is decomposed. It is omitted in ongoing analysis.

Characteristic polynomial for (2.2) is

$P(\lambda)=\sum_{j=0}^{5} C_{j} \lambda^{j}=0$ 
coefficients are

$C_{5}=1$,

$C_{4}=\theta_{B} \varepsilon \cos ^{2} \Theta+\varepsilon \sin ^{2} \Theta+\theta_{A} \varepsilon$,

$C_{3}=\theta_{A} \theta_{B} \varepsilon^{2} \cos ^{2} \Theta+\theta_{A} \varepsilon^{2} \sin ^{2} \Theta-4 \lambda_{A} \lambda_{B}+4$,

$C_{2}=4 \theta_{A} \varepsilon \lambda_{B}-4 \lambda_{A} \lambda_{B} \varepsilon \sin ^{2} \Theta+4 \varepsilon \sin ^{2} \Theta-4 \lambda_{A} \theta_{B} \varepsilon \cos ^{2} \Theta$,

$C_{1}=4 \theta_{A} \lambda_{B} \varepsilon^{2} \sin ^{2} \Theta-16 \lambda_{A} \lambda_{B}$,

$C_{0}=-16 \lambda_{A} \lambda_{B} \varepsilon \sin ^{2} \Theta$.

Necessary stability conditions are $C_{j}>0$. This is obviously true for $C_{4}$ and $C_{5}$. $C_{0}>0$ if $\lambda_{A} \lambda_{B}<0$, so $C$ is either maximum or minimum moment of inertia. Remaining $C_{j}$ are also positive in this case. Further investigation of polynomial (2.3) is complicated by overburdened coefficients $C_{j}$. Note that equations (2.2) have the form

$\dot{\mathbf{x}}=\mathbf{A}_{0} \mathbf{x}+\varepsilon \mathbf{A}_{1} \mathbf{x}$

where $\mathbf{x}=\left(\omega_{1}, \omega_{2}, \omega_{3}, \beta, \gamma\right)$. Parameter $\varepsilon$ may be considered small since magnetic control system frequently employs weak magnetorquers. Write characteristic exponents in the same form, $\lambda_{j}=\mu_{j}+\varepsilon \eta_{j}$. Zero approximation $(\varepsilon=0)$ exponents are easily found,

$\mu_{1,2}= \pm 2 \sqrt{-\lambda_{A} \lambda_{B}} i$

$\mu_{3}=0$,

$\mu_{4,5}= \pm 2 i$.

Here $i$ is imaginary unit. Here necessary stability condition $\lambda_{A} \lambda_{B}<0$ was used. Characteristic polynomial coefficients are $C_{j}=A_{j}+\varepsilon B_{j}$,

$$
\begin{array}{ll}
A_{5}=1, & B_{5}=0, \\
A_{4}=0, & B_{4}=\theta_{B} \cos ^{2} \Theta+\sin ^{2} \Theta+\theta_{A}, \\
A_{3}=-4 \lambda_{A} \lambda_{B}+4, & B_{3}=0, \\
A_{2}=0, & B_{2}=4 \theta_{A} \lambda_{B}-4 \lambda_{A} \lambda_{B} \sin ^{2} \Theta+4 \sin ^{2} \Theta-4 \lambda_{A} \theta_{B} \cos ^{2} \Theta, \\
A_{1}=-16 \lambda_{A} \lambda_{B}, & B_{1}=0, \\
A_{0}=0 . & B_{0}=-16 \lambda_{A} \lambda_{B} \sin ^{2} \Theta .
\end{array}
$$

Terms of the order of $O\left(\varepsilon^{2}\right)$ are omitted. Characteristic polynomial is

$$
P(\mu+\varepsilon \eta)=\sum_{j=0}^{5}\left(A_{j}+\varepsilon B_{j}\right)(\mu+\varepsilon \eta)^{j}=0 .
$$


Taking into account that $\mu_{j}$ satisfy (2.3) first order approximation becomes $\eta_{j}=-\frac{\sum_{k=0}^{5} \mu_{j}^{k} B_{k}}{\sum_{k=0}^{4}(k+1) \mu_{j}^{k} A_{k+1}}$.

Taking into account coefficients and zero order approximation this yields $\eta_{1,2}=-\frac{\theta_{A} \lambda_{B}\left(1+\lambda_{A}\right)+\theta_{B} \cos ^{2} \Theta \lambda_{A}\left(\lambda_{B}-1\right)}{2\left(\lambda_{A} \lambda_{B}+1\right)}$, $\eta_{3}=-\sin ^{2} \Theta$ $\eta_{4,5}=-\frac{\theta_{A}\left(1-\lambda_{B}\right)+\theta_{B} \cos ^{2} \Theta\left(\lambda_{A}+1\right)}{2\left(\lambda_{A} \lambda_{B}+1\right)}$.

Due to inertia properties $\left|\lambda_{A}\right|<1$ and hence $\eta_{4,5}<0$. However $\eta_{1,2}<0$ if and only if $\lambda_{B}>0, \lambda_{A}<0$. So only rotation around the maximum axis of inertia is asymptotically stable.

\section{Stabilization accuracy}

Simplified dipole model provides general qualitative result - stable rotation with double angular velocity. Direct dipole model is more accurate and has one important peculiarity. Geomagnetic induction vector rotation is non-uniform. We use this property to refine both direction of spinning satellite axis in inertial space and this rotation rate. Poincare method for periodical solutions of differential equation is used for this purpose [6].

\subsection{Satellite on near equatorial orbit}

Satellite motion is represented with respect to $O_{a} S_{1} S_{2} S_{3}$ frame. Small parameter is orbit inclination $i$. Geomagnetic induction vector is decomposed retaining the second order of small parameter

$\mathbf{B}_{S}=\frac{\mu_{e}}{r^{3}}\left(\begin{array}{l}0 \\ 0 \\ 1\end{array}\right)+\frac{\mu_{e}}{r^{3}}\left(\begin{array}{c}-3 / 2 \sin 2 u \\ 1-3 \sin ^{2} u \\ 0\end{array}\right) i+\frac{\mu_{e}}{r^{3}}\left(\begin{array}{c}0 \\ 0 \\ -1 / 2\end{array}\right) i^{2}$.

Equation of motion have the form

$\dot{\mathbf{x}}=\mathbf{f}(\mathbf{x})+i \mathbf{g}_{1}(\mathbf{x})+i^{2} \mathbf{g}_{2}(\mathbf{x})$

where $\mathbf{x}=\left(\omega_{1}, \omega_{2}, \omega_{3}, \alpha, \beta, \gamma\right)$. Periodical solution of (3.1) is also of the form $\mathbf{x}=\mathbf{x}_{0}+i \mathbf{x}_{1}+i^{2} \mathbf{x}_{2}$. 
Note that $\mathbf{x}_{0}$ is (3.1) solution for $i=0$ (generating periodical solution). Asymptotical stability of solution $\mathbf{x}_{0}=(\Omega u, 0,0,0,0, \Omega)$ may be proven just like in previous section, so this is the only periodical solution. Angular velocity $\Omega$ is arbitrary. Substituting (3.2) into (3.1) we obtain for different orders of magnitude of $i$

$$
\begin{aligned}
i^{0} & \dot{\mathbf{x}}_{0}=\mathbf{f}\left(\mathbf{x}_{0}\right), \\
i^{1} & \dot{\mathbf{x}}_{1}=\left.\frac{\partial \mathbf{f}}{\partial \mathbf{x}}\right|_{\mathbf{x}_{0}} \mathbf{x}_{1}+\mathbf{g}_{1}\left(\mathbf{x}_{0}\right), \\
i^{2} & \dot{\mathbf{x}}_{2}=\left.\frac{1}{2} \frac{\partial \mathbf{f}}{\partial \mathbf{x}}\right|_{\mathbf{x}_{0}} \mathbf{x}_{2}+\left.\frac{1}{2} \mathbf{x}_{1}^{T} \frac{\partial^{2} \mathbf{f}}{\partial \mathbf{x}^{2}}\right|_{\mathbf{x}_{0}} \mathbf{x}_{1}+\left.\frac{\partial \mathbf{g}_{1}}{\partial \mathbf{x}}\right|_{\mathbf{x}_{0}} \mathbf{x}_{1}+\mathbf{g}_{2}\left(\mathbf{x}_{0}\right) .
\end{aligned}
$$

First order approximation equations are decomposed into two systems

$$
\dot{\mathbf{z}}_{1}=\mathbf{F} \mathbf{z}_{1}+\varepsilon\left(\begin{array}{c}
\theta_{A}[3 / 2(\Omega-2) \sin (\Omega-2) u-1 / 2 \sin \Omega u] \\
\theta_{B}[3 / 2(\Omega-2) \cos (\Omega-2) u-1 / 2 \cos \Omega u] \\
0 \\
0
\end{array}\right),
$$

$\dot{\omega}_{3}^{(1)}=0, \dot{\alpha}^{(1)}=\omega_{3}^{(1)}$

where $\mathbf{z}_{1}=\left(\omega_{1}^{(1)}, \quad \omega_{2}^{(1)}, \quad \beta^{(1)}, \gamma^{(1)}\right), \varepsilon=k\left(\mu_{e} / r^{3}\right)^{2} / C \omega_{0}$ is a new parameter.

$$
\mathbf{F}=\left(\begin{array}{cccc}
-\varepsilon \theta_{A} & \Omega \lambda_{A} & 0 & -\delta \Omega \theta_{A} \\
\Omega \lambda_{B} & -\varepsilon \theta_{B} & \delta \Omega \theta_{B} & 0 \\
1 & 0 & 0 & \Omega \\
0 & 1 & -\Omega & 0
\end{array}\right)
$$

First order does not allow the exact value $\Omega$ to be determined. Second order approximation equations are used,

$$
\begin{aligned}
& \mathbf{z}_{2}=\mathbf{F} \mathbf{z}_{2}+\mathbf{G}(u) \mathbf{z}_{1}, \\
& \dot{\omega}_{3}^{(2)}=3 \varepsilon(\Omega-1) \cos 2 u-5 \Omega+9, \dot{\alpha}^{(2)}=\omega_{3}^{(2)}
\end{aligned}
$$

where $\mathbf{G}(u)$ is periodical matrix. Equation for $\omega_{3}^{(2)}$ provides condition of periodical solution existence $\Omega=1.8$. Satellite spins a little slower than in the simplified dipole magnetic field.

Equilibria perturbation is characterized by equations (3.3). Unperturbed system equilibrium position is asymptotically stable. General solution of (3.3) asymptotically tends to zero. Partial solution has governs system behavior. It may be written as $\mathbf{z}_{p}=\mathbf{a}_{\Omega} \cos \Omega u+\mathbf{b}_{\Omega} \sin \Omega u+\mathbf{a}_{\Omega-2} \cos (\Omega-2) u+\mathbf{b}_{\Omega-2} \sin (\Omega-2) u$. 
Constant vectors $\mathbf{a}$ and $\mathbf{b}$ are obtained by substituting (3.4) into (3.3),

$$
\begin{aligned}
& \left(\begin{array}{l}
\mathbf{a}_{\Omega} \\
\mathbf{b}_{\Omega}
\end{array}\right)=\left(\begin{array}{cc}
\Omega \mathbf{E}_{4 \times 4} & -\mathbf{F} \\
-\mathbf{F} & -\Omega \mathbf{E}_{4 \times 4}
\end{array}\right)^{-1}\left(\begin{array}{l}
\mathbf{q}_{\Omega} \\
\mathbf{p}_{\Omega}
\end{array}\right), \\
& \left(\begin{array}{l}
\mathbf{a}_{\Omega-2} \\
\mathbf{b}_{\Omega-2}
\end{array}\right)=\left(\begin{array}{cc}
(\Omega-2) \mathbf{E}_{4 \times 4} & -\mathbf{F} \\
-\mathbf{F} & -(\Omega-2) \mathbf{E}_{4 \times 4}
\end{array}\right)^{-1}\left(\begin{array}{l}
\mathbf{q}_{\Omega-2} \\
\mathbf{p}_{\Omega-2}
\end{array}\right)
\end{aligned}
$$

where

$$
\begin{aligned}
& \mathbf{q}_{\Omega}=\left(\begin{array}{llll}
0, & -1 / 2 \theta_{B} \varepsilon, & 0, & 0
\end{array}\right), \mathbf{p}_{\Omega}=\left(\begin{array}{llll}
-1 / 2 \theta_{B} \varepsilon, & 0, & 0, & 0
\end{array}\right), \\
& \mathbf{q}_{\Omega-2}=-3(\Omega-2) \mathbf{q}_{\Omega}, \mathbf{p}_{\Omega-2}=-3(\Omega-2) \mathbf{p}_{\Omega} \text {. }
\end{aligned}
$$

Calculations lead to

$\mathbf{a}_{\Omega}=\left(\begin{array}{llll}0, & 0, & 0, & -1 / 2\end{array}\right), \mathbf{b}_{\Omega}=\left(\begin{array}{llll}0, & 0, & 1 / 2, & 0\end{array}\right)$.

$\mathbf{q}_{\Omega-2}$ and $\mathbf{p}_{\Omega-2}$ calculation involves block matrix inverse

$$
\left(\begin{array}{cc}
\kappa \mathbf{E}_{4 \times 4} & -\mathbf{F} \\
-\mathbf{F} & -\kappa \mathbf{E}_{4 \times 4}
\end{array}\right)^{-1}=\left(\begin{array}{cc}
\frac{1}{\kappa}\left(\mathbf{E}_{4 \times 4}-\left(\mathbf{E}_{4 \times 4}+\kappa^{2} \mathbf{F}^{-2}\right)^{-1}\right) & -\left(\mathbf{E}_{4 \times 4}+\kappa^{2} \mathbf{F}^{-2}\right)^{-1} \mathbf{F}^{-1} \\
-\mathbf{F}^{-1}\left(\mathbf{E}_{4 \times 4}+\kappa^{2} \mathbf{F}^{-2}\right)^{-1} & -\kappa \mathbf{F}^{-1}\left(\mathbf{E}_{4 \times 4}+\kappa^{2} \mathbf{F}^{-2}\right)^{-1} \mathbf{F}^{-1}
\end{array}\right) .
$$

Since $\kappa=\Omega-2 \ll 1(3.5)$ becomes

$$
\left(\begin{array}{cc}
\kappa \mathbf{E}_{4 \times 4} & -\mathbf{F} \\
-\mathbf{F} & -\kappa \mathbf{E}_{4 \times 4}
\end{array}\right)^{-1} \approx\left(\begin{array}{cc}
\kappa^{2} \mathbf{F}^{-2} & -\mathbf{F}^{-1} \\
-\mathbf{F}^{-1} & -\kappa \mathbf{F}^{-2}
\end{array}\right) .
$$

Therefore

$$
\mathbf{z}_{1}=\left(\begin{array}{c}
0 \\
0 \\
1 / 2 \sin \Omega u \\
-1 / 2 \cos \Omega u
\end{array}\right)-3\left(\kappa^{3} \mathbf{F}^{-2} \mathbf{q}_{\Omega}-\kappa \mathbf{F}^{-1} \mathbf{p}_{\Omega}\right) \cos \kappa u+3\left(\kappa \mathbf{F}^{-1} \mathbf{q}_{\Omega}+\kappa^{2} \mathbf{F}^{-2} \mathbf{p}_{\Omega}\right) \sin \kappa u .
$$

Since $\kappa$ is small solutions $\beta^{(1)}$ and $\gamma^{(1)}$ are mainly governed by $\mathbf{a}_{\Omega}$ and $\mathbf{b}_{\Omega}$, $\beta^{(1)} \approx 1 / 2 \sin \Omega u, \gamma^{(1)} \approx-1 / 2 \cos \Omega u$.

Rotation axis deviates by the angle $\Delta \gamma_{33}$ from the orbit normal,

$$
\begin{aligned}
\Delta \gamma_{33} & =\operatorname{acos}[\cos (1 / 2 i \sin 1.8 u) \cos (-1 / 2 i \cos 1.8 u)]= \\
& =2 \operatorname{asin} \sqrt{\frac{1-\cos (1 / 2 i \sin 9 / 5 u) \cos (-1 / 2 i \cos 9 / 5 u)}{2}} \approx \\
& \approx 2 \operatorname{asin} 1 / 4 i \approx 1 / 2 i .
\end{aligned}
$$

Therefore angle between the maximum moment of inertia axis and normal to the orbit is approximately equal to the half of the inclination. 


\subsection{Near polar satellite}

Near polar orbits are of more interest for magnetically actuated satellites. Consider this case and new small parameter $\delta=\pi / 2-i$. Geomagnetic induction vector is decomposed as

$\mathbf{B}_{S}=\frac{\mu_{e}}{r^{3}}\left(\begin{array}{c}-3 / 2 \sin 2 u \\ 1-3 \sin ^{2} u \\ 0\end{array}\right)+\frac{\mu_{e}}{r^{3}}\left(\begin{array}{l}0 \\ 0 \\ 1\end{array}\right) \delta+\frac{\mu_{e}}{2 r^{3}}\left(\begin{array}{c}-3 / 2 \sin 2 u \\ 1-3 \sin ^{2} u \\ 0\end{array}\right) \delta^{2}$.

Generating solution equations are divided into two systems. Out of plane motion allows trivial solution $\beta=\gamma=0, \omega_{1}=\omega_{2}=0$ again. Rotation around maximum moment of inertia persist. Rotation rate becomes varying. This rate is governed by the equation

$\dot{\omega}_{3}+f_{1}(u) \omega_{3}=f_{2}(u)$

where

$f_{1}(u)=\varepsilon\left(1+3 \sin ^{2} u\right)$,

$f_{2}(u)=3 \varepsilon\left(1+\sin ^{2} u\right)$.

Solution of (3.7) is (assume $u(0)=0$ ),

$\omega_{3}=e^{-F(u)}\left(\omega_{3}(0)+\int_{0}^{u} f_{2}(x) e^{F(x)} d x\right)$

where

$F(u)=\int_{0}^{u} f_{1}(x) d x=\varepsilon\left(\frac{5}{2} u-\frac{3}{4} \sin 2 u\right)$.

Integral in (3.8) poses a problem. Rewrite it as

$g(u)=\int_{0}^{u} f_{2}(x) e^{F(x)} d x=\varepsilon \int_{0}^{u} 2 e^{\varepsilon\left(\frac{5}{2} x-\frac{3}{4} \sin 2 x\right)} d x+\varepsilon \int_{0}^{u}\left(1+3 \sin ^{2} x\right) e^{\varepsilon\left(\frac{5}{2} x-\frac{3}{4} \sin 2 x\right)} d x=$ $=e^{\varepsilon\left(\frac{5}{2} u-\frac{3}{4} \sin 2 u\right)}+2 \varepsilon \int_{0}^{u} e^{\varepsilon \frac{5}{2} x} e^{-\varepsilon \frac{3}{4} \sin 2 x} d x$.

Hence

$\omega_{3}=e^{-F(u)}\left(\omega_{3}(0)+e^{F(u)}+2 \varepsilon \int_{0}^{u} e^{\varepsilon \frac{5}{2} x} e^{-\frac{3}{4} \sin 2 x} d x\right)$.

Note that $-1 \leq \sin 2 x \leq 1$. Angular velocity is bounded by following relations

$e^{-\varepsilon\left(\frac{5}{2} u-\frac{3}{4} \sin 2 u\right)} \omega_{3}(0)+1+\frac{4}{5} e^{-\frac{3}{4} \varepsilon} e^{\frac{3}{4} \varepsilon \sin 2 u} \leq \omega_{3} \leq e^{-\varepsilon\left(\frac{5}{2} u-\frac{3}{4} \sin 2 u\right)} \omega_{3}(0)+1+\frac{4}{5} e^{\frac{3}{4} \varepsilon} e^{\frac{3}{4} \varepsilon \sin 2 u}$. 
Influence of the second term in (3.9) vanishes as argument of latitude rises. This term may be omitted given appropriate time interval and

$$
\begin{aligned}
& g(u)=\int_{0}^{u} f_{2}(x) e^{F(x)} d x \approx 3 \varepsilon \int_{0}^{u}\left(1+\sin ^{2} x\right) e^{5 / 2 \varepsilon x} d x= \\
& =6 \varepsilon\left[\frac{e^{5 / 2 \varepsilon u} \sin u}{25 \varepsilon^{2}+16}(5 \varepsilon \sin u-4 \cos u)+\frac{1}{\varepsilon}\left(\frac{8}{125 \varepsilon^{2}+80}+\frac{1}{5}\right)\left(e^{5 / 2 \varepsilon u}-1\right)\right] .
\end{aligned}
$$

Substituting into (3.9) and (3.8) brings angular velocity of rotation around the maximum moment of inertia

$$
\omega_{3} \approx e^{-\varepsilon\left(\frac{5}{2}-\frac{3}{4} \sin 2 u\right)}\left(\omega_{3}(0)+6 \varepsilon\left[\frac{e^{5 / 2 \varepsilon u} \sin u}{25 \varepsilon^{2}+16}(5 \varepsilon \sin u-4 \cos u)+\frac{1}{\varepsilon}\left(\frac{8}{125 \varepsilon^{2}+80}+\frac{1}{5}\right)\left(e^{5 / 2 \varepsilon u}-1\right)\right]\right) .
$$

Angle $\alpha$ in generating solution is found integrating (3.11).

First order equations are

$\dot{\mathbf{x}}_{1}=\mathbf{F}(u) \mathbf{x}_{1}+\mathbf{g}(u)$

where $\quad F_{i j}=F_{i j}\left(\omega_{3}, \varphi(2 u-\alpha), \varphi(4 u-2 \alpha), \varphi(2 u-2 \alpha), \varphi(2 \alpha), \varphi(2 u)\right), \quad \varphi \quad$ are trigonometric functions (sine and cosine). They appear in relations for $\mathbf{F}$ components linearly (exact expression of $\mathbf{F}$ can be found in appendix). These equations cannot be solved. Approximate solution of the first order equations is found using Poincare method again. Small parameter is $\varepsilon$ (control torque value). Solution of the equations of motion is represented as

$\mathbf{x}=\mathbf{x}_{0}+\delta \mathbf{x}_{1}=\mathbf{x}_{0}+\delta\left(\mathbf{y}_{0}+\varepsilon \mathbf{y}_{1}\right)$,

$\mathbf{x}_{0}=\left(0,0, \omega_{3}(u), \alpha(u), 0,0\right)$, matrix $\mathbf{F}$ is $\mathbf{F}(u)=\mathbf{F}_{0}+\varepsilon \mathbf{F}_{1}(u)$. Generating solution $\mathbf{y}_{0}$ and first order approximation $\mathbf{y}_{1}$ are found from equations

$$
\dot{\mathbf{z}}_{0}=\mathbf{F}_{0} \mathbf{z}_{0},
$$

$\dot{\mathbf{z}}_{1}=\mathbf{F}_{0} \mathbf{z}_{1}+\mathbf{F}_{1}(u) \mathbf{z}_{0}+\mathbf{g}_{1}(u)$

and

$$
\begin{aligned}
& \dot{\omega}_{3}^{(0)}=0, \dot{\alpha}^{(0)}=\omega_{3}^{(0)}, \\
& \dot{\omega}_{3}^{(1)}=(3 / 2 \cos 2 u-5 / 2) \omega_{3}^{(0)}, \dot{\alpha}^{(1)}=0
\end{aligned}
$$

where $\mathbf{z}_{1}=\left(\omega_{1}^{(1)}, \omega_{2}^{(1)}, \quad \beta^{(1)}, \quad \gamma^{(1)}\right)$.

Independent equations yield $\omega_{3}^{(0)}=0, \omega_{3}^{(1)}, \alpha^{(0)}, \alpha^{(1)}$ are constant.

Expressions in (3.12)-(3.13) contain $\omega_{3}(u)$. We use (3.11) to find its approximate value. This expression is decomposed assuming small parameter $\varepsilon$ and sufficient time interval, 
$\omega_{3}=9 / 5+3 / 5 \varepsilon \sin 2 u$.

This leads to

$\mathbf{F}_{0}=\left(\begin{array}{cccc}0 & 9 / 5 \lambda_{A} & 0 & 0 \\ 9 / 5 \lambda_{B} & 0 & 0 & 0 \\ 1 & 0 & 0 & 9 / 5 \\ 0 & 1 & -9 / 5 & 0\end{array}\right), \mathbf{g}_{1}(u)=\left(\begin{array}{c}\theta_{A}(3 / 10 \sin 0.2 u-9 / 10 \sin 1.8 u) \\ \theta_{B}(-3 / 10 \cos 0.2 u-9 / 10 \cos 1.8 u) \\ 0 \\ 0\end{array}\right)$.

Matrix $\mathbf{F}_{1}(u)$ is written as $\mathbf{F}_{1}(u)=\mathbf{G}_{1}(u)+\mathbf{G}_{2}(u)$,

$\mathbf{G}_{1}(u)=\left(\begin{array}{cccc}-\frac{5}{4} \theta_{A}-\frac{1}{8} \theta_{A} \cos 3.6 u & \frac{1}{8} \theta_{A} \sin 3.6 u & -\frac{9}{40} \theta_{A} \sin 3.6 u & -\frac{9}{4} \theta_{A}-\frac{9}{40} \theta_{A} \cos 3.6 u \\ \frac{1}{8} \theta_{B} \sin 3.6 u & -\frac{5}{4} \theta_{B}+\frac{1}{8} \theta_{B} \sin 3.6 u & \frac{9}{4} \theta_{B}-\frac{9}{40} \theta_{B} \cos 3.6 u & \frac{9}{40} \theta_{B} \sin 3.6 u \\ 0 & 0 & 0 & 0 \\ 0 & 0 & 0 & 0\end{array}\right)$,

components $G_{2 i j}=G_{2 i j}(\varphi(0.2 u), \varphi(0.4 u), \varphi(1.6 u), \varphi(2 u))$ (exact expression for $\mathbf{F}_{1}(u)$ can be found in appendix).

Generating solution for (3.12) is

$\mathbf{y}_{0}=\left(\begin{array}{c}A \cos \lambda u+B \sin \lambda u \\ -A \mu \sin \lambda u+B \mu \cos \lambda u \\ D \cos 1.8 u+E \sin 1.8 u+B \chi_{2} \cos \lambda u+A \chi_{4} \sin \lambda u \\ -D \sin 1.8 u+E \cos 1.8 u+A \chi_{3} \cos \lambda u+B \chi_{1} \sin \lambda u\end{array}\right)$

where $\lambda=1.8 \sqrt{-\lambda_{A} \lambda_{B}}, \mu=\sqrt{-\lambda_{B} / \lambda_{A}}, \chi_{i}=\chi_{i}(\lambda, \mu), A, B, D, E$ are constant. They are found from the existence conditions of periodical solution of equations (3.13). This implies that heterogeneous part $\mathbf{F}_{1}(u) \mathbf{z}_{0}+\mathbf{g}_{1}(u)$ does not contain frequencies $\lambda$ and 1.8 (Eigen frequency of $\mathbf{F}_{0}$ ). $\mathbf{F}_{1}(u)$ and $\mathbf{g}_{1}(u)$ contain only rational numbers so $A=B=0$. Constants $D, E$ are found from absence of frequency 1.8 in $\mathbf{G}_{1}(u) \mathbf{z}_{0}+\mathbf{g}_{1}(u)$ (matrix $\mathbf{G}_{2}(u)$ may be omitted) so $E=0, D=4 / 9$.

Approximate solution of equations of motions is $\omega_{1}=\omega_{2}=0$, $\beta=4 / 9(\pi / 2-i) \cos 1.8 u, \gamma=-4 / 9(\pi / 2-i) \sin 1.8 u$,

$\omega_{3}$ is governed by one of the expressions (3.10), (3.11), (3.14), $\alpha$ can be found integrating $\omega_{3}$. Axis of the maximum moment of inertia deviates from the orbit normal by the angle 


$$
\Delta \gamma_{33}=\operatorname{acos}[\cos (4 / 9(\pi / 2-i) \cos 1.8 u) \cos (-4 / 9(\pi / 2-i) \sin 1.8 u)] .
$$

Decomposing this analogous to (3.6) provides $\Delta \gamma_{33} \approx 4 / 9(\pi / 2-i)$. Deviation is slightly less for near-polar orbits.

\section{Numerical simulation}

Numerical simulation was carried out with following parameters:

- $\quad$ Inertia tensor $\mathbf{J}=(1.4,1.6,2.0) \mathrm{kg} \cdot \mathrm{m}^{2}$

- $\quad$ Orbit inclination $75^{\circ}$, altitude $750 \mathrm{~km}$

- Initial conditions: attitude angles $50^{\circ}$, angular velocity components $1.1 \omega_{0}$

- Control gain $5 \cdot 10^{5} \mathrm{~N} \cdot \mathrm{m} \cdot \mathrm{s} / \mathrm{T}^{2} \cdot \mathrm{kg} \cdot \mathrm{m}$ (approximately $\varepsilon \approx 0.11$ )

Fourth order Runge-Kutta with constant time step $1 \mathrm{~s}$ was used. Fig. 2 brings simulation results for the right dipole model. Fig. 3 presents analogous result for inclined dipole model.
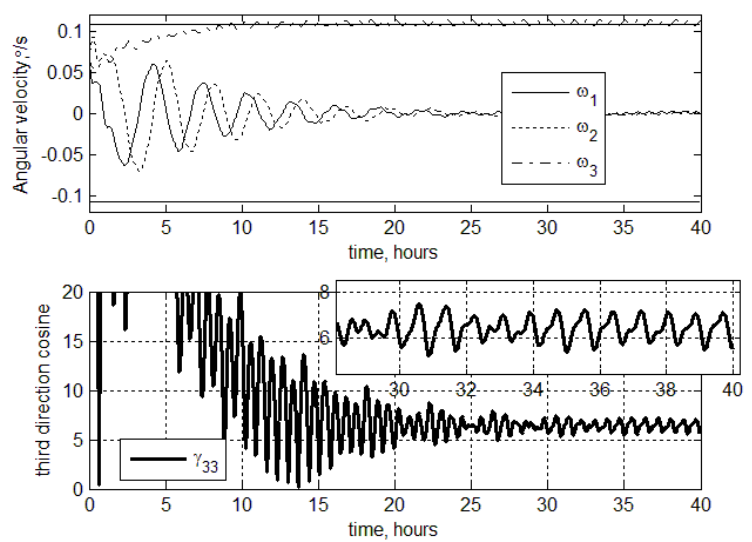

Fig. 2. Near polar orbit, right dipole model
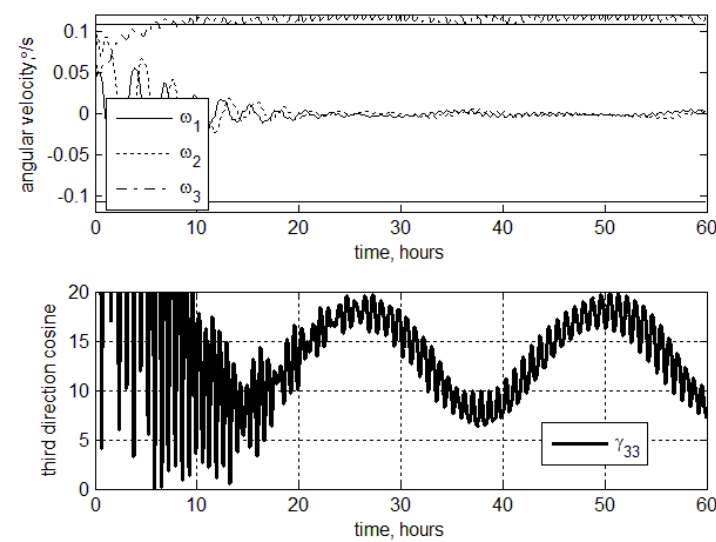

Fig. 3. Near polar orbit, inclined dipole model

Fig. 2 verifies analytical results. Axis of maximum moment of inertia is stabilized with $5.5-7^{\circ}$ accuracy while analytical expression provides $6.5^{\circ}$. Inclined dipole model observes almost three times worse accuracy. Horizontal line on figures corresponds to angular velocity $1.8 \omega_{0}$. Near equatorial orbit yields same resultsю Figures 4 and 5 provide results for orbit inclination $60^{\circ}$. Right dipole model is used in both cases. Gravitational torque is taken into account for Fig. 5. 

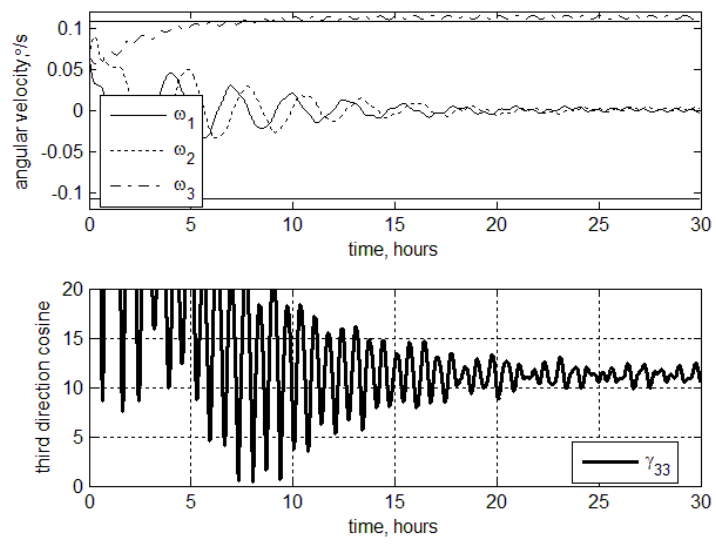

Fig. 4. Inclination $60^{\circ}$, right dipole model
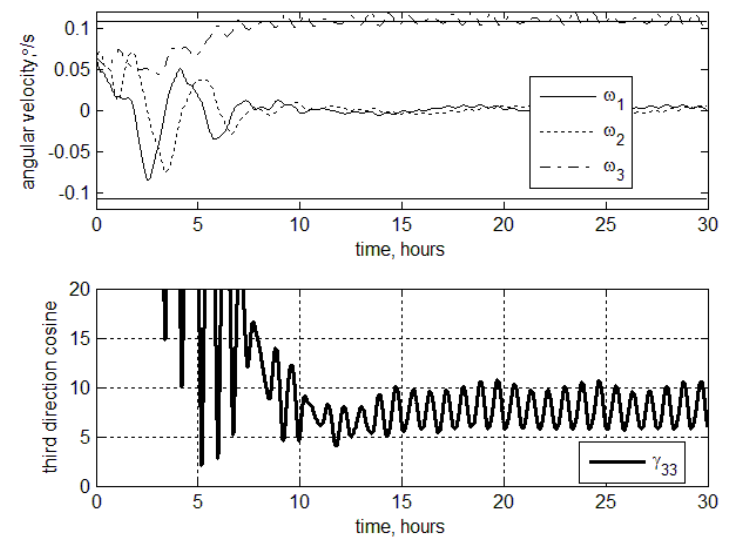

Fig. 5. Inclination $60^{\circ}$, gravitational torque

Arbitrary orbits that far from near polar or near equatorial yield spinning axis pointing accuracy of about $10-12^{\circ}$. Gravitational torque acts as a restoring one. Moreover its magnitude is almost comparable with the control magnetic torque. As a result stabilization accuracy is better.

\section{Conclusion}

Magnetically actuated satellite with «-Bdot» damping algorithm is considered. Slow motion of the satellite is studied. Rotation around the maximum moment of inertia is proved to be asymptotically stable in simplified dipole geomagnetic field. Rotation velocity is found in right dipole field. Maximum moment of inertia axis attitude in inertial space is found.

\section{Bibliography}

1. Ovchinnikov M.Y. et al. Investigation of the effectiveness of an algorithm of active magnetic damping // Cosm. Res. 2012. V. 50, № 2. pp. 170-176.

2. Ovchinnikov M.Y., Roldugin D.S. Dual-spin satellite motion in magnetic and gravitational fields // Keldysh Institute Preprints. 2015. № 22. 20 p.

3. Stickler A.C. A Magnetic Control System for Attitude Acquisition // Ithaco, Inc., Rep. N 90345. 1972.

4. Stickler A.C., Alfriend K.T. Elementary Magnetic Attitude Control System // J. Spacecr. Rockets. 1976. V. 13, № 5. pp. 282-287.

5. Pichuzhkina A.V., Roldugin D.S. Geomagnetic field models for satellite angular motion // Keldysh Institute Preprints. 2016. № 87. 25 p.

6. Malkin I.G. Some problems in the theory of nonlinear oscillations. Oak Ridge: U.S. Atomic Energy Commission, Technical Information Service, 1959. 589 p. 


\section{Appendix. Some relations}

$\mathbf{F}(u)=\left(\begin{array}{cccccc}-\varepsilon \theta_{A}\left(\frac{5}{4}+f_{1}\right) & \lambda_{A} \omega_{3}+\varepsilon \theta_{A} f_{2} & 0 & 0 & -\varepsilon \theta_{A} \omega_{3} f_{2} & -\varepsilon \theta_{A} f_{3} \\ \lambda_{B} \omega_{3}+\varepsilon \theta_{B} f_{2} & -\varepsilon \theta_{B}\left(\frac{5}{4}-f_{1}\right) & 0 & 0 & \varepsilon \theta_{B} f_{3} & \varepsilon \theta_{B} \omega_{3} f_{2} \\ 0 & 0 & \varepsilon\left(\frac{3}{2} \cos 2 u-\frac{5}{2}\right) & 0 & 0 & 0 \\ 0 & 0 & 1 & 0 & 0 & 0 \\ 1 & 0 & 0 & 0 & 0 & \omega_{3} \\ 0 & 1 & 0 & 0 & -\omega_{3} & 0\end{array}\right)$,

$f_{1}=\frac{9}{8} \cos 2(\alpha-2 u)+\frac{1}{8} \cos 2 \alpha-\frac{3}{4} \cos 2(\alpha-u)-\frac{3}{4} \cos 2 u$,

$f_{2}=-\frac{3}{4} \sin 2(\alpha-u)+\frac{9}{8} \sin 2(\alpha-2 u)+\frac{1}{8} \sin 2 \alpha$,

$f_{3}=\frac{3}{4} \omega_{3} \cos 2 u-\frac{3}{2} \cos 2 u-\frac{5}{4} \omega_{3}-\frac{3}{4} \omega_{3} \cos 2(\alpha-u)+$

$$
+\frac{9}{8} \omega_{3} \cos 2(\alpha-2 u)+\frac{1}{8} \omega_{3} \cos 2 \alpha+\frac{9}{2} \text {. }
$$

$\mathbf{F}_{1}(u)=\left(\begin{array}{cccc}-\theta_{A}\left(\frac{5}{4}+g_{1}\right) & \frac{3}{5} \lambda_{A} \sin 2 u+\theta_{A} g_{2} & -\frac{9}{5} \theta_{A} g_{2} & -\theta_{A} g_{3} \\ \frac{3}{5} \lambda_{B} \sin 2 u+\theta_{B} g_{2} & -\theta_{B}\left(\frac{5}{4}-g_{1}\right) & \theta_{B} g_{3} & \frac{9}{5} \theta_{B} g_{2} \\ 0 & 0 & 0 & \frac{3}{5} \sin 2 u \\ 0 & 0 & -\frac{3}{5} \sin 2 u & 0\end{array}\right)$,

$g_{1}=\frac{9}{8} \cos 0.4 u+\frac{1}{8} \cos 3.6 u-\frac{3}{4} \cos 1.6 u-\frac{3}{4} \cos 2 u$,

$g_{2}=-\frac{3}{4} \sin 1.6 u-\frac{9}{8} \sin 0.4 u+\frac{1}{8} \sin 3.6 u$,

$g_{3}=\frac{9}{4}-\frac{3}{20} \cos 2 u-\frac{27}{20} \cos 1.6 u+\frac{81}{40} \omega_{3} \cos 0.4 u+\frac{9}{40} \cos 3.6 u$. 